\title{
EFEITOS REDISTRIBUTIVOS E DETERMINANTES DE RECEBIMENTO DO ICMS ECOLÓGICO PELOS MUNICÍPIOS MINEIROS
}

\author{
Luciany Lima Fernandes' \\ Alexandre Bragança Coelho² \\ Elaine Aparecida Fernandes ${ }^{3}$ \\ João Eustáquio de Lima ${ }^{4}$
}

\section{Resumo}

Esse artigo teve como objetivo analisar a mudança na distribuição do ICMS aos municípios mineiros pela introdução do critério ecológico. Procurouse também avaliar os determinantes da probabilidade de os municípios pertencerem à categoria de receptores do ICMS Ecológico. Os resultados obtidos mostram que este recurso como mecanismo de distribuição beneficia mais intensamente os municípios menores, mas seu impacto é pequeno pelo reduzido valor de seu percentual (1\%). Além disso, o impacto do ICMS Ecológico para esses municípios vem perdendo força nos últimos anos com o aumento do numero de municípios recebedores, o que é preocupante em termos do incentivo a novos municípios que queiram se adequar às normas de recebimento deste recurso.

Palavras-Chaves: ICMS Ecológico, Minas Gerais, Modelo Logit.

Classificação JEL: Q50, H23, C35 


\section{INTRODUÇÃO}

O Imposto sobre Circulação de Mercadorias e Serviços (ICMS) é um imposto estadual sobre o valor adicionado de bens e serviços. A Constituição de 1988 determinou que $25 \%$ das receitas do ICMS deveriam ser transferidas dos estados para os municípios que os compõem. A Constituição também estipulou que, no mínimo, $75 \%$ desta receita transferida deveria ser distribuída de acordo com o valor adicionado gerado em cada município ${ }^{5}$ e o restante seria distribuído de acordo com critérios de alocação que cada estado achasse mais conveniente ao seu caso específico. Os indicadores usados normalmente eram baseados no tamanho da população, área do município e produção primária local.

Em 1991, o critério ecológico passou a ser utilizado para distribuir parte destes recursos no Estado do Paraná. Esta experiência pioneira originou-se da reivindicação dos municípios que sentiam que suas economias eram prejudicadas pelas restrições de uso do solo, originadas por serem mananciais de abastecimento para municípios vizinhos ou por integrarem Unidades de Conservação. Dessa forma, o poder público estadual sentiu a necessidade de reformular os critérios de distribuição do ICMS, favorecendo estes municípios com recursos adicionais. Apesar de o ICMS Ecológico nascer sob a égide da compensação, sua característica mais importante é constituir-se num mecanismo de incentivo à conservação ambiental, representando uma promissora alternativa na composição dos instrumentos de política pública para a conservação ambiental no Brasil (LOUREIRO, 2002).

Em Minas Gerais ${ }^{6}$, o ICMS Ecológico foi criado através da Lei Complementar Estadual número 12.040/95, chamada de "Lei Robin Hood". O modelo mineiro, além do critério ambiental, inclui outros como: patrimônio cultural, educação, produção de alimentos, número de habitantes por município, 50 municípios mais populosos, receita própria municipal, saúde e área geográfica (MINAS GERAIS, 1995).

Para cada município, o valor adicionado mede a produção econômica do município, traduzida pela diferença entre o somatório das notas fiscais de venda e o somatório das notas fiscais de compra.

6 Além do Estado do Paraná e de Minas Gerais, outros Estados como: São Paulo (1993), Rondônia (1996), Amapá (1996), Rio Grande do Sul (1997), Mato Grosso (2000), Mato Grosso do Sul (2000), Pernambuco (2000) e Tocantins (2002) implantaram o ICMS Ecológico, com pequenas diferenças entre eles. 
Para o critério ambiental, a lei destina $0,5 \%$ do ICMS aos municípios que possuam unidades de conservação e $0,5 \%$ aos municípios que possuam sistema de tratamento ou disposição final de lixo urbano que atenda pelo menos $70 \%$ da população, ou sistema de tratamento de esgoto sanitário que atenda pelo menos $50 \%$ da população ${ }^{7}$ (NUNES, 2003).

O ICMS Ecológico foi introduzido através da diminuição do peso do critério de valor adicionado ${ }^{8}$. Ao mudar os critérios de distribuição, muitos municípios com Unidades de Conservação aumentaram sua receita. Entretanto, é importante observar que muitos municípios perderam receita pelo fato de o critério de valor adicionado ter perdido importância ${ }^{9}$. Além disso, para municípios considerados pequenos ou de pouca expressão econômica, o repasse ecológico representou mais de $20 \%$ do repasse total feito pelo estado, sendo uma fonte importante de recursos (GRIEG-GRAN, 2000). Diante desse contexto, torna-se relevante analisar, de forma detalhada, como o critério ecológico repercute na receita dos municípios mineiros, procurando compreender como se distribuem as perdas e os ganhos dos municípios.

Este trabalho tem como objetivo geral analisar a mudança na distribuição do ICMS aos municípios mineiros pela introdução do critério ecológico. Além disso, pretende-se avaliar também os determinantes da probabilidade de os municípios receberem o ICMS Ecológico. Apesar de as regras para os municípios se qualificarem a receber o ICMS ecológico serem claras e estarem contidas na Lei "Robin Hood", como salientado anteriormente, o que se busca com essa análise é entender como as características dos municípios afetam a probabilidade de eles pertencerem a categoria dos municípios passíveis de receber este recurso. Assim, um modelo de escolha binária (logit) será utilizado para analisar como variáveis importantes como tamanho da população, área, escolaridade da população, mesorregião a que município pertence, entre outras, afetam esta probabilidade.

Este artigo contém, além dessa introdução, mais três seções. A próxima descreve a metodologia utilizada na análise dos dados. Na terceira,

\footnotetext{
Ver anexo A para entender como este montante é distribuído para cada município.

Ver anexo B.

Grieg-Gran (2000), ao analisar os dados de Minas Gerais em 1998, concluiu que 86 municípios com Unidades de Conservação foram beneficiados com aumento de receita pela introdução do ICMS Ecológico, enquanto 38 municípios perderam receita pela diminuição do critério de valor adicionado.
} 
são discutidos os principais resultados encontrados, e a última descreve as conclusões obtidas.

\section{METODOLOGIA}

\subsection{Distribuição do ICMS Ecológico}

A introdução do ICMS Ecológico foi feita pela redução de outros critérios, especialmente o critério de valor adicionado. Dessa forma, é necessário, em primeiro lugar, analisar o efeito da introdução do ICMS ecológico na porcentagem total de ICMS que cada município recebe, o chamado índice consolidado. Entretanto, mudanças no índice consolidado são resultado de vários fatores, e não só do ICMS ecológico. Segundo Grieg-Gran (2000), é necessário examinar como a introdução de um novo critério e, em particular, o critério ecológico afeta a arrecadação dos municípios, de forma a indicar os ganhadores e os perdedores neste processo. Uma comparação foi realizada entre índices atuais dos municípios mineiros e aqueles que resultariam nos seguintes cenários:

1. Aplicação de um peso de $100 \%$ para o valor adicionado, isto é, o ICMS seria redistribuído completamente na base do valor adicionado.

2. Situação pré-Robin Hood com a eliminação do critério ecológico e de outros critérios introduzidos pela Lei Robin Hood (população, área geográfica, educação, produção de alimentos, patrimônio cultural, receita própria municipal e saúde) e um aumento de $14,71 \%{ }^{10}$ no peso aplicado ao valor adicionado.

3. Situação pré-Robin Hood e um peso de $1 \%$ aplicado ao critério ecológico.

A construção destes 3 cenários objetivou separar o efeito do critério ecológico de outros fatores. Dessa forma, uma desagregação das mudanças no

10 Este valor refere-se à porcentagem destinada à Lei Robin Hood nos anos de 1998 a 2007. No ano de 1997, o valor é de 10,04\%. 
índice consolidado por cada fator (valor adicionado, índice ecológico, outros critérios) foi feito, procurando isolar o efeito de cada um, mostrando qual o impacto do ICMS ecológico na receita dos municípios de Minas Gerais.

\subsection{Determinantes de pertencer à categoria de receptores do ICMS Ecológico}

Para analisar os determinantes da probabilidade de pertencer à categoria de receptores do ICMS Ecológico, será utilizado o modelo logit, que se baseia na utilização da Função de Distribuição Acumulada Logística (FDAL) (GUJARATI, 2006).

A expressão para este modelo é dada pela equação (1):

$$
P_{i}=E\left(Y_{i}=1\right)=F\left(X_{i} \beta\right)=\frac{1}{1+e^{-\left(X_{i} \beta\right)}}
$$

em que $\mathrm{P}_{\mathrm{i}}$ representa a probabilidade de o município i receber o ICMS Ecológico; $\mathrm{X}_{\mathrm{i}} \beta$ é o valor da regressão estimada para cada município, considerando-se as variáveis explicativas que foram incluídas no modelo, ou seja, $X_{i} \beta=\beta_{0}+\beta_{1} X_{1 i}+\ldots+\beta_{K} X_{K i}$, em que os $\beta$ 's são os parâmetros estimados e os X's, as variáveis independentes consideradas representando as características deste município; i são os municípios que compõem o total de observações da amostra utilizada; e "e" é a base de logaritmos naturais, que é aproximadamente igual a 2,718.

Reescrevendo-se (1) tem-se:

$$
P_{i}=\frac{1}{1+e^{-Z_{i}}}=\frac{e^{Z}}{1+e^{Z}}
$$

em que $Z_{i}=X_{i} \beta$.

Se $P_{i}$ é a probabilidade do município receber o ICMS Ecológico, então $\left(1-\mathrm{P}_{\mathrm{i}}\right)$ é a probabilidade de ele não receber esse imposto, o qual pode ser expressa por:

$$
1-P_{i}=\frac{1}{1+e^{Z_{i}}}
$$

A relação entre $\mathrm{P}_{\mathrm{i}}$ e $\left(1-\mathrm{P}_{\mathrm{i}}\right)$ pode ser escrita da seguinte forma: 


$$
\frac{P_{i}}{1-P_{i}}=\frac{1+e^{Z_{i}}}{1+e^{-Z_{i}}}=e^{Z_{i}}
$$

em que $\frac{P_{i}}{1-P_{i}}$ é a razão de chances a favor de receber o ICMS Ecológico, ou seja, a razão da probabilidade de que um município receba o ICMS Ecológico contra a probabilidade de ele não receber.

Para linearizar os parâmetros na expressão (4), aplica-se o logaritmo natural em ambos os lados da equação, obtendo-se:

$$
\begin{aligned}
& L_{i}=\ln \left(\frac{P_{i}}{1-P_{i}}\right)=Z_{i} \quad \text { ou } \\
& L_{i}=\ln \left(\frac{P_{i}}{1-P_{i}}\right)=X_{i} \beta
\end{aligned}
$$

A equação (5) representa o logaritmo da razão de chances, conhecido como modelo logit $\left(\mathrm{L}_{\mathrm{i}}\right)$. O modelo (1) é estimado pelo método da máxima verossimilhança.

Substituindo $\mathrm{X}_{\mathrm{i}} \beta$ pelas variáveis explicativas utilizadas neste trabalho, tem-se o seguinte modelo:

$$
\begin{aligned}
& =\beta_{1}+\beta_{2} V A_{i}+\beta_{3} A R E A_{i}+\beta_{4} A N A L F+\beta_{5} P O_{i}+\alpha_{1} D_{1 i}+\alpha_{2} D_{2 i}+ \\
& \alpha_{3} D_{3 i}+\alpha_{4} D_{4 i}+\alpha_{5} D_{5 i}+\alpha_{6} D_{6 i}+\alpha_{7} D_{7 i}+\alpha_{8} D_{8 i}+\alpha_{9} D_{9 i}+\alpha_{10} D_{10 i}+\alpha_{11} D_{11 i}+{ }_{i}
\end{aligned}
$$

A definição das variáveis presentes no modelo (6) é a seguinte:

- $\quad \mathrm{VA}_{\mathrm{i}}$ é o ICMS recebido pelo município i através do critério valor adicionado (em reais). O sinal esperado do coeficiente dessa variável é negativo, pois quanto maior ICMS recebido através do critério valor adicionado, maior a atividade econômica do município, o que, dados os padrões históricos de crescimento econômico, significa invariavelmente maior degradação ambiental e, portanto, menor probabilidade de recebimento do ICMS Ecológico. 
- $\quad$ AREA $_{i}$ é a área total do município i (em hectares). Espera-se que esta variável apresente sinal positivo, pois quanto maior a área de um município, maiores as chances de possuir em seu território áreas protegidas.

- ANALF $_{\mathrm{i}}$ é a taxa de analfabetismo de 15 anos ou mais. Espera-se que esta variável apresente sinal negativo, pois quanto maior a escolaridade e menor a taxa de analfabetismo, maior a expectativa de que haja pressão da sociedade por uma maior preocupação ambiental das administrações municipais.

- $\quad \mathrm{PO}_{\mathrm{i}}$ é o tamanho da população. Espera-se que o sinal desta variável seja negativo, pois quanto maior a população de um município, maior a pressão sobre os recursos naturais e menor a probabilidade de recebimento do ICMS Ecológico.

- $\quad \mathrm{D}_{\mathrm{ji}}(\mathrm{j}=1,2, \ldots, 11)$ são variáveis Dummies, sendo que elas se referem a localização do município, com o grupo-base (dummy omitida na estimação) representando Zona da Mata $\left(\mathrm{D}_{1 \mathrm{i}}\right.$, mesorregião Campos das Vertentes; $\mathrm{D}_{2 \mathrm{i}}$, Central Mineira; $\mathrm{D}_{3 \mathrm{i}}$, Jequitinhonha; $\mathrm{D}_{4 \mathrm{i}}$, metropolitana de Belo Horizonte; $\mathrm{D}_{5 \mathrm{i}}$, Noroeste de Minas; $\mathrm{D}_{6}$, Norte de Minas; $\mathrm{D}_{7 \mathrm{i}}$, Oeste de Minas; $\mathrm{D}_{8 \mathrm{i}}$, Sul e Sudoeste de Minas; $\mathrm{D}_{9 \mathrm{i}}$, Triângulo Mineiro e Alto do Paranaíba; $\mathrm{D}_{10 \mathrm{i}}$, Vale do Mucuri; $\mathrm{D}_{11 \text { i }}$, Vale do Rio Doce ${ }^{11}$ ). Espera-se que as variáveis $\mathrm{D}_{1}, \mathrm{D}_{2}, \mathrm{D}_{3}, \mathrm{D}_{5}, \mathrm{D}_{6}, \mathrm{D}_{7}, \mathrm{D}_{8}, \mathrm{D}_{9}, \mathrm{D}_{10}$ apresentem um sinal negativo e $\mathrm{D}_{4}$ e $\mathrm{D}_{11}$ tenham um sinal positivo ${ }^{12}$.

- $\varepsilon_{\mathrm{i}}$ é o erro aleatório.

Para calcular o efeito marginal sobre $\mathrm{P}_{\mathrm{i}}$ de uma variável explicativa contínua em cada ponto, é preciso derivar a equação (1) em relação à variável explicativa de interesse.

Utilizando a regra do quociente do cálculo diferencial, tem-se:

$$
\frac{\partial P_{i}}{\partial X_{K i}}=\beta_{K} \cdot \frac{1}{\left(1+e^{-\left(X_{i} \beta\right)}\right)} \cdot \frac{\left(e^{-\left(X_{i} \beta\right)}\right)}{\left(1+e^{-\left(X_{i} \beta\right)}\right)}
$$

\footnotetext{
Estas 12 regiões são aquelas utilizadas pelo IBGE.

12 Em 2005, as mesorregiões Metropolitana de Belo Horizonte, Vale do Rio Doce e Zona da Mata possuíam, respectivamente, $65,71 \%, 52,94 \%$ e 50,35\% de seus municípios recebendo o ICMS Ecológico. Todas as demais regiões apresentaram percentuais bem inferiores a estes, o que determinaria a expectativa em relação ao sinal das variáveis dummies.
} 
Observando que o $2^{\circ}$ termo à direita da expressão (7) é $\mathrm{P}_{\mathrm{i}}$ e o terceiro é (1- $\left.\mathrm{P}_{\mathrm{i}}\right)$, tem-se que:

$$
\frac{\partial P_{i}}{\partial X_{K i}}=\beta_{K} \cdot P_{i} \cdot\left(1-P_{i}\right)
$$

A expressão (8) significa, em pontos percentuais, a variação na probabilidade de um município pertencer à categoria de receptores do ICMS Ecológico, dada uma mudança na variável independente $\mathrm{X}$, mantendo-se as demais constantes.

Com relação ao efeito marginal sobre as variáveis binárias, ele é calculado da seguinte forma:

$$
E M_{x k}=P\left[\left(y_{i}=1 / x_{k}=1\right)\right]-P\left[\left(y_{i}=1 / x_{k}=0\right)\right]
$$

em que: $E M_{x k}$ é o efeito marginal da variável binária $x_{k}$; $P\left[\left(y_{i}=1 / x_{k}=1\right)\right]$ é a probabilidade de o município pertencer à categoria de receptores do ICMS Ecológico quando $x_{k}=1 ; P\left[\left(y_{i}=1 / x_{k}=0\right)\right]$ é a probabilidade de o município pertencer à categoria de receptores do ICMS Ecológico quando $x_{k}=0$.

\subsection{Fonte dos dados}

Esse trabalho utilizou dados anuais, no período de 1997 a 2007, para as seguintes variáveis: o valor do ICMS distribuído a todos os municípios; o valor do ICMS Ecológico repassado aos municípios e o ICMS distribuído aos municípios através do critério valor adicionado fiscal. Esses dados foram obtidos junto a Fundação João Pinheiro. Para as variáveis: população, Produto Interno Bruto e área dos municípios, utilizaram-se dados obtidos no site do Instituto Brasileiro de Geografia e Estatística (IBGE). A variável Produto Interno Bruto foi obtida no período de 1999 a 2005 devido à disponibilidade de dados. Já a taxa de analfabetismo de 15 anos ou mais foi obtida no site do Instituto Nacional de Estudos e Pesquisas Educacionais (INEP) no ano de 2005. Todos os valores monetários foram deflacionados pelo Índice Geral de Preços Disponibilidade Interna IGPDI, da Fundação Getúlio Vargas. 


\section{RESULTADOS E DISCUSSÃO}

\subsection{Efeitos redistributivos do ICMS Ecológico}

Para determinar quais foram os municípios ganhadores e perdedores depois da criação da Lei Robin Hood, construíram-se três cenários hipotéticos e esses foram comparados com a situação atual, utilizando a metodologia vista na seção 2.1. A construção destes três cenários foi feita para separar o efeito do critério ecológico dos outros critérios, verificando assim qual foi o impacto do ICMS Ecológico nas receitas dos municípios mineiros. Classificaram-se os municípios por tamanho e os resultados encontram-se na Tabela 1.

Com relação à população, classificaram-se os municípios em pequenos, médios, médios a grandes e grandes, com populações, respectivamente, na faixa de até 10.000 habitantes, de 10.001 a 50.000 habitantes, de 50.001 a 100.000 habitantes e de 100.001 habitantes em diante ${ }^{13}$. Os resultados encontrados estão na Tabela 1.

Os resultados médios obtidos na comparação entre o primeiro cenário (em que o valor adicionado é o único critério) e a Lei Robin Hood mostram que apenas $3,22 \%$ dos municípios com população até 10.000 habitantes estariam em melhor situação que o cenário atual. Já com a Lei Robin Hood (cenário atual), a porcentagem aumenta para 96,78\%. Para os municípios médios, $18,76 \%$ estariam melhor com o primeiro cenário e $81,24 \%$ estariam melhores com a Lei Robin Hood. Para os municípios de médio a grandes, $64,23 \%$ optariam pelo primeiro cenário e $35,77 \%$ optariam pelo cenário atual. E para os municípios grandes, 79,23\% estariam em melhores condições com o primeiro cenário e apenas $20,77 \%$ estariam melhores no cenário atual. Estes resultados podem ser vistos também na Figura 1. Assim, a Lei Robin Hood com todos os seus critérios favorece realmente os municípios menores.

\footnotetext{
3 Observa-se, no ano de 2007, que existiam 493 municípios considerados pequenos, 296 considerados médios, 38 considerados médios a grandes e 26 municípios considerados grandes (IBGE, 2007).
} 
Tabela 1. Porcentagem de municípios mineiros, segundo a população, em situação favorável a cada um dos cenários, média 1997 a 2007

\begin{tabular}{cccccc}
\hline \multirow{2}{*}{ ano } & & \multicolumn{4}{c}{ população } \\
& cenários & até 10.000 & $10.001 \mathrm{a}$ & $50.001 \mathrm{a}$ & 100.001 \\
& & 50.000 & 100.000 & em diante \\
\hline \multirow{3}{*}{ média } & $1^{\circ}$ cenário & 3,22 & 18,76 & 64,23 & 79,23 \\
& atual & 96,78 & 81,24 & 35,77 & 20,77 \\
& $2^{\circ}$ cenário & 4,91 & 19,91 & 53,47 & 66,81 \\
& atual & 95,09 & 80,09 & 46,53 & 33,19 \\
& $3^{\circ}$ cenário & 7,18 & 20,31 & 47,92 & 57,12 \\
& atual & 92,82 & 79,69 & 52,08 & 42,88 \\
\hline
\end{tabular}

Fonte: Resultados da Pesquisa.

O $2^{\circ}$ cenário é aquele em que ocorre a situação pré-Robin Hood, existindo somente os critérios: valor adicionado, municípios mineradores, cota mínima, Mateus Leme e Mesquita ${ }^{14}$. Os valores médios encontrados na Tabela 1 mostram que 4,91\% dos municípios pequenos ganhariam com o $2^{\circ}$ cenário e 95,09\% ganhariam com a Lei Robin Hood. Já para os municípios médios, 19,91\% estariam em melhores condições com o $2^{\circ}$ cenário e $80,09 \%$ com o cenário atual. Para os municípios médios a grandes, 53,47\% ganhariam com o $2^{\circ}$ cenário e 46,53\% ganhariam com a Lei Robin Hood. Para os municípios grandes, $66,81 \%$ ganhariam com o $2^{\circ}$ cenário e $33,19 \%$ ganhariam com o cenário atual. Os resultados confirmam assim que a Lei Robin Hood beneficia os municípios menores, pois diminui o peso do critério de valor adicionado na distribuição de ICMS.

${ }_{14}$ Ver anexo $B$ para as porcentagens de cada critério. 


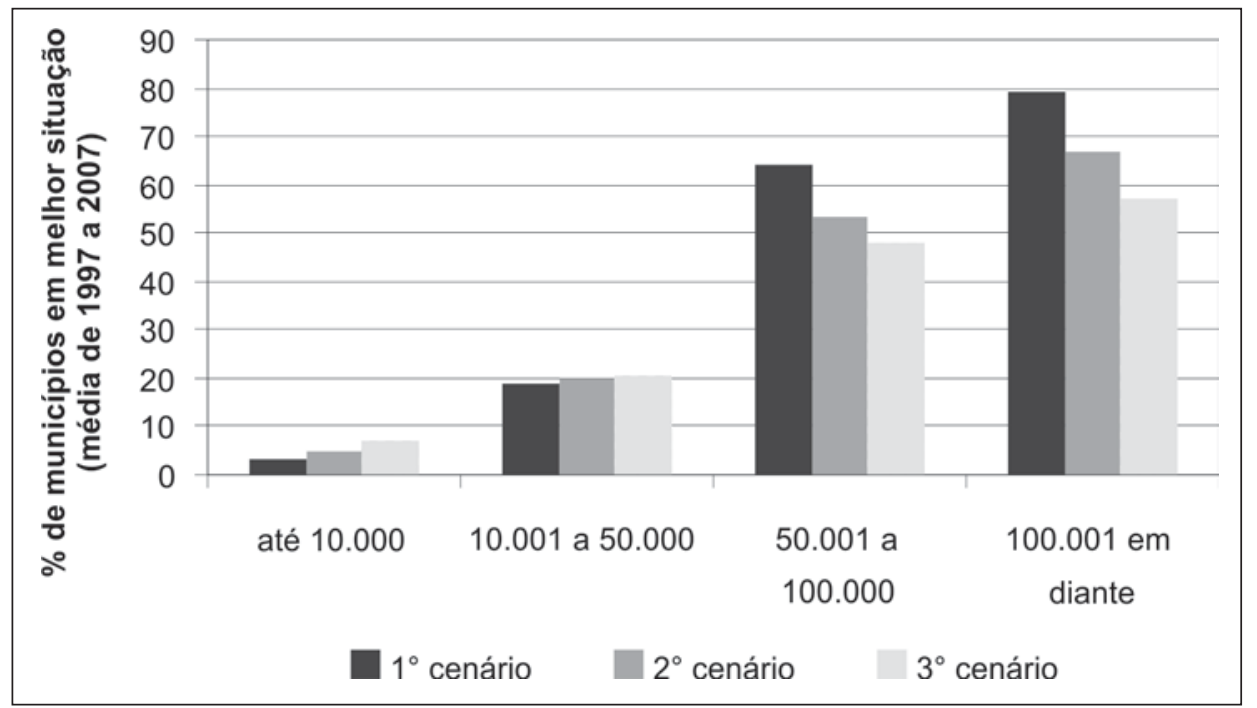

Fonte: Resultados da pesquisa.

Figura 1. Comparação entre os três cenários e a Lei Robin Hood, utilizando a população, média de 1997 a 2007.

O $3^{\circ}$ cenário indica a situação pré-Robin Hood com um peso de $1 \%$ aplicado ao critério ecológico, mostrando o impacto do ICMS Ecológico sem os outros critérios presentes nessa lei. Os valores médios encontrados na Tabela 1 mostram que 7,18\% dos municípios pequenos ganhariam com o $3^{\circ}$ cenário e $92,82 \%$ ganhariam com a Lei Robin Hood. Já para os municípios médios, 20,31\% estariam em melhores condições com o $3^{\circ}$ cenário e 79,69\% com o cenário atual. Dos municípios médios a grandes, $47,92 \%$ ganhariam com o $3^{\circ}$ cenário e $52,08 \%$ ganhariam com a Lei Robin Hood. Para os municípios grandes, $57,12 \%$ ganhariam com o $3^{\circ}$ cenário e 42,88\% ganhariam com o cenário atual. Assim, a Lei Robin Hood beneficia realmente os municípios menores e o ICMS Ecológico sozinho, sem os outros critérios da Lei Robin Hood, tem um impacto pequeno no ganho obtido pelos municípios.

A Tabela 2 mostra o número de municípios em situação favorável em cada um dos cenários. Em relação aos valores médios obtidos nesta tabela, tem-se que 111 municípios ganhariam com o $1^{\circ}$ cenário e 742 ganhariam com a Lei Robin Hood. Grieg-Gran (2000), considerando o cenário "atual" em janeiro de 1998, obteve que 740 municípios ganharam com a situação 
atual comparado ao cenário em que somente o valor adicionado foi distribuído, resultado parecido com o que foi obtido neste trabalho. Portanto, observa-se, em todo o período analisado, que a Lei Robin Hood, ao retirar peso do critério de valor adicionado, beneficiou a maior parte dos municípios mineiros, especialmente os municípios menores.

Tabela 2. Número de municípios mineiros em situação favorável a cada um dos cenários no período 1997 a 2007

\begin{tabular}{ccccccc}
\hline ano & $1^{\circ}$ cenário & atual & $2^{\circ}$ cenário & atual & $3^{\circ}$ cenário & atual \\
\hline 1997 & 120 & 733 & 131 & 722 & 202 & 651 \\
1998 & 116 & 737 & 135 & 718 & 164 & 689 \\
1999 & 106 & 747 & 124 & 729 & 133 & 720 \\
2000 & 103 & 750 & 109 & 744 & 107 & 746 \\
2001 & 112 & 741 & 117 & 736 & 110 & 743 \\
2002 & 104 & 749 & 110 & 743 & 110 & 743 \\
2003 & 106 & 747 & 107 & 746 & 105 & 748 \\
2004 & 111 & 742 & 109 & 744 & 109 & 744 \\
2005 & 114 & 739 & 111 & 742 & 110 & 743 \\
2006 & 116 & 737 & 114 & 739 & 113 & 740 \\
2007 & 117 & 736 & 111 & 742 & 112 & 741 \\
média* $^{*}$ & 111 & 742 & 116 & 737 & 125 & 728 \\
\hline
\end{tabular}

Fonte: Resultados da Pesquisa.

* Estes valores são arredondados.

Na comparação realizada entre o $2^{\circ}$ cenário e o atual, os valores médios obtidos mostram que 116 municípios estariam em melhor situação com o $2^{\circ}$ cenário e 737 municípios com o cenário atual (Tabela 2). Isto mostra a importância de todos os critérios contidos na Lei Robin Hood. Segundo Grieg-Gran (2000), o número de municípios que ganhou com essa lei foi de 725, comparando com a situação pré-Robin Hood. Assim, em todo o período analisado, observa-se que a Lei Robin Hood foi realmente efetiva em seus objetivos, beneficiando os municípios menores.

Os valores médios obtidos na comparação entre o $3^{\circ}$ cenário e o atual mostram que 125 municípios ganhariam com o $3^{\circ}$ cenário e 728 ganhariam com o cenário atual, mostrando que os outros critérios são mais importantes que o ICMS Ecológico na distribuição do montante entre os municípios. Observa-se também que como o percentual do ICMS Ecológico permaneceu fixo e o número de municípios cresceu, a fatia destinada a cada município 
foi ficando cada vez menor, o que se reflete no número decrescente de municípios em situação favorável o $3^{\circ}$ cenário.

Para verificar o impacto do ICMS Ecológico sem os outros critérios presentes na Lei Robin Hood, optou-se pela comparação entre o $2^{\circ}$ cenário (situação pré-Robin Hood) e o $3^{\circ}$ cenário (situação pré-Robin Hood com um peso de $1 \%$ aplicado ao critério ecológico). Os resultados encontrados estão presentes na Tabela 3 e na Figura 2. Para estes resultados, optou-se também em separar os municípios por tamanho.

Tabela 3. Porcentagem dos municípios, segundo a população, em situação favorável a cada um dos cenários, período de 1997 a 2007

\begin{tabular}{|c|c|c|c|c|c|}
\hline \multirow[b]{2}{*}{ ano } & \multirow[b]{2}{*}{ cenários } & \multicolumn{4}{|c|}{ população } \\
\hline & & até 10.000 & $\begin{array}{c}10.001 \mathrm{a} \\
50.000\end{array}$ & $\begin{array}{c}50.001 \mathrm{a} \\
100.000\end{array}$ & $\begin{array}{c}100.001 \\
\text { em diante }\end{array}$ \\
\hline \multirow{2}{*}{1997} & $2^{\circ}$ cenário & 47,02 & 46,01 & 41,67 & 55,00 \\
\hline & $3^{\circ}$ cenário & 52,98 & 53,99 & 58,33 & 45,00 \\
\hline \multirow{2}{*}{1998} & $2^{\circ}$ cenário & 47,89 & 40,00 & 42,86 & 61,90 \\
\hline & $3^{\circ}$ cenário & 52,11 & 60,00 & 57,14 & 38,10 \\
\hline \multirow{2}{*}{1999} & $2^{\circ}$ cenário & 47,61 & 44,85 & 44,44 & 59,09 \\
\hline & $3^{\circ}$ cenário & 52,39 & 55,15 & 55,56 & 40,91 \\
\hline \multirow[b]{2}{*}{2000} & $2^{\circ}$ cenário & 83,14 & 81,62 & 88,89 & 91,30 \\
\hline & $3^{\circ}$ cenário & 16,86 & 18,38 & 11,11 & 8,70 \\
\hline \multirow{2}{*}{2001} & $2^{\circ}$ cenário & 82,05 & 78,55 & 86,49 & 91,30 \\
\hline & $3^{\circ}$ cenário & 17,95 & 21,45 & 13,51 & 8,70 \\
\hline \multirow{2}{*}{2002} & $2^{\circ}$ cenário & 77,82 & 77,62 & 84,21 & 87,50 \\
\hline & $3^{\circ}$ cenário & 22,18 & 22,38 & 15,79 & 12,50 \\
\hline \multirow{2}{*}{2003} & $2^{\circ}$ cenário & 73,15 & 72,92 & 83,33 & 96,15 \\
\hline & $3^{\circ}$ cenário & 26,85 & 27,08 & 16,67 & 3,85 \\
\hline \multirow{2}{*}{2004} & $2^{\circ}$ cenário & 69,01 & 68,84 & 86,84 & 96,15 \\
\hline & $3^{\circ}$ cenário & 30,99 & 31,16 & 13,16 & 3,85 \\
\hline \multirow{2}{*}{2005} & $2^{\circ}$ cenário & 67,51 & 67,27 & 86,84 & 92,31 \\
\hline & $3^{\circ}$ cenário & 32,49 & 32,73 & 13,16 & 7,69 \\
\hline \multirow[b]{2}{*}{2006} & $2^{\circ}$ cenário & 67,39 & 65,70 & 82,50 & 88,89 \\
\hline & $3^{\circ}$ cenário & 32,61 & 34,30 & 17,50 & 11,11 \\
\hline \multirow{2}{*}{2007} & $2^{\circ}$ cenário & 68,97 & 70,61 & 78,95 & 88,46 \\
\hline & $3^{\circ}$ cenário & 31,03 & 29,39 & 21,05 & 11,54 \\
\hline \multirow{2}{*}{ média } & $2^{\circ}$ cenário & 66,51 & 64,91 & 73,37 & 82,55 \\
\hline & $3^{\circ}$ cenário & 33,49 & 35,09 & 26,63 & 17,45 \\
\hline
\end{tabular}

Fonte: Resultados da Pesquisa. 
O que se destaca nesta Tabela é a reversão do impacto do ICMS Ecológico sobre os municípios pequenos e médios (até 100.000 habitantes). No inicio da implantação desse recurso, como poucos municípios estavam aptos a recebê-lo, o aumento de receita para cada município recebedor era considerável, fazendo com que a maioria de pequenos e médios se beneficiasse (maior porcentagem de municípios em situação favorável no $3^{\circ}$ cenário). Isso se modifica radicalmente a partir do ano 2000, quando o crescimento do numero de municípios recebedores diminui a fatia individual de cada um, causando a reversão do numero de beneficiados com o terceiro cenário. Assim, a situação pré-Robin Hood, sem o ICMS Ecológico, seria melhor para a maior parte dos pequenos e médios municípios mineiros em termos de recebimento de recursos repassados pelo Estado.

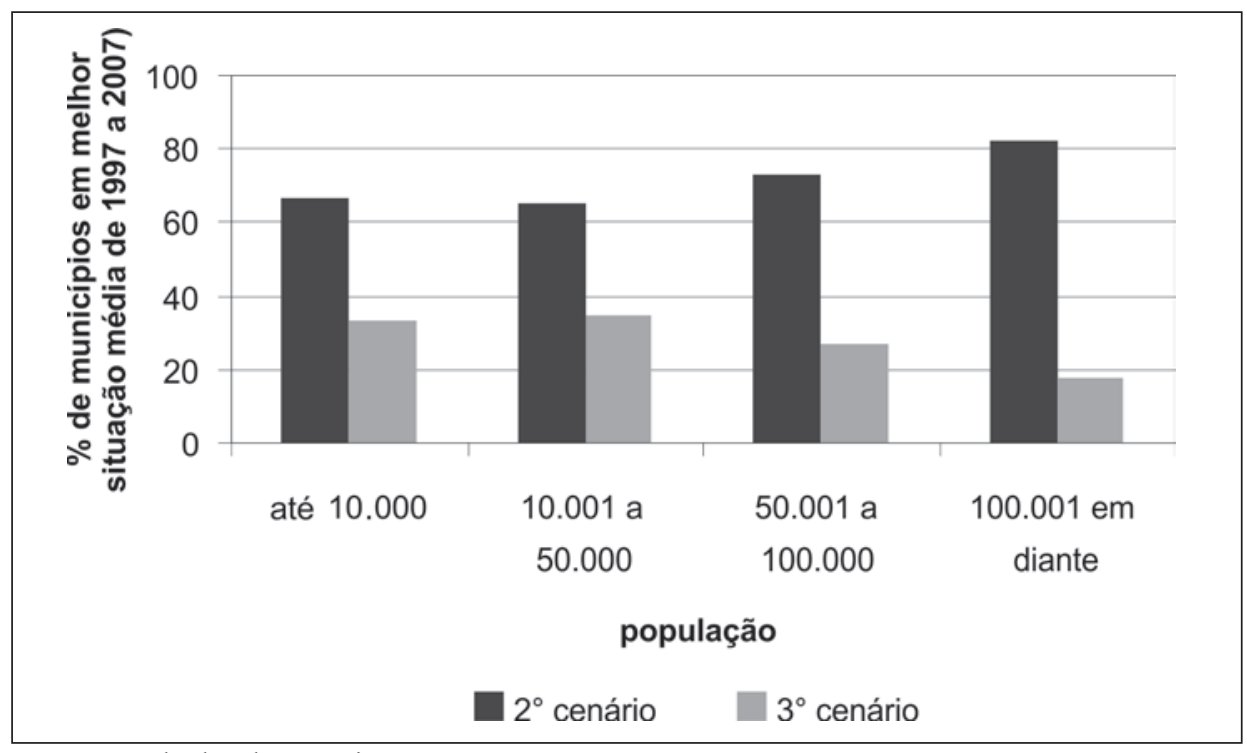

Fonte: Resultados da pesquisa.

Figura 2. Comparação entre o $2^{\circ}$ cenário e o $3^{\circ}$ cenário, utilizando a população, média de 1997 a 2007.

A Figura 3 apresenta os resultados do $2^{\circ}$ e $3^{\circ}$ cenários para os municípios que efetivamente recebem o ICMS Ecológico. Pode-se observar que em todo o período analisado, a maior parte dos municípios que recebem o ICMS Ecológico seria beneficiada com o $3^{\circ}$ cenário, como era de se esperar, embora 
existam municípios (cerca de 30\% deles) que perderiam com este cenário, mesmo recebendo o ICMS Ecológico, pois perderiam receita pela diminuição do peso do critério de valor adicionado. Portanto, do grupo de municípios que recebem o ICMS Ecológico, existem mais ganhadores do que perdedores.

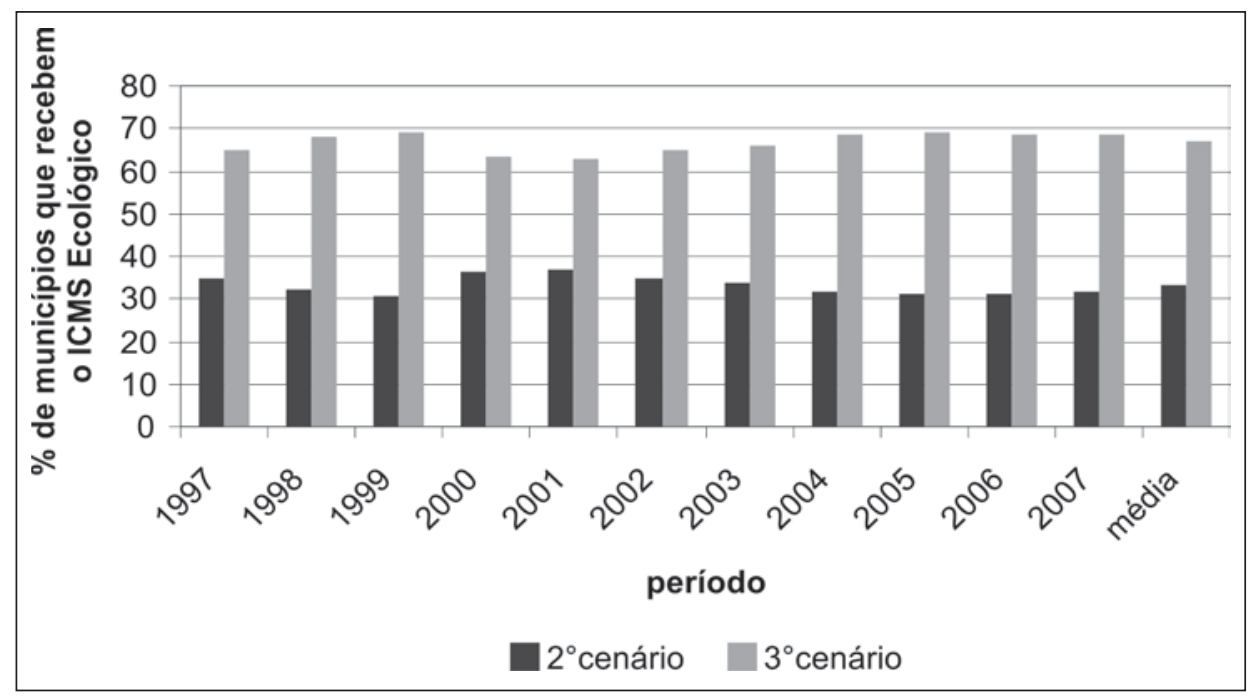

Fonte: Resultados da Pesquisa

Figura 3. Porcentagem dos municípios que recebem o ICMS Ecológico em cada um dos cenários, período 1997 a 2007.

Dessa forma, com relação aos efeitos redistributivos do ICMS Ecológico, pode-se concluir que ele realmente beneficia mais intensamente os municípios menores e com baixos níveis de renda, mas seu impacto é pequeno pelo reduzido valor de seu percentual (1\%). Além disso, o impacto do ICMS Ecológico para esses municípios vem perdendo força nos últimos anos com o aumento do numero de municípios recebedores, o que é preocupante em termos do incentivo a novos municípios que queiram se adequar às normas de recebimento deste recurso. Além disso, como há atualmente mais perdedores do que ganhadores com o ICMS Ecológico entre os municípios com baixo nível de renda e menores, pode haver pressão dos representantes políticos desses municípios junto ao Governo estadual para mudar ou revogar este dispositivo, o que poderia reverter importantes ganhos na gestão ambiental municipal em Minas Gerais. 


\subsection{Os determinantes da probabilidade de pertencer à categoria de receptores do ICMS Ecológico}

Conforme proposto na metodologia, utilizou-se o modelo logit para verificar quais os determinantes da probabilidade de pertencer à categoria de receptores do ICMS Ecológico. Estimou-se o modelo cujos resultados encontram-se na Tabela 4. Pode-se notar que oito das dezesseis variáveis são significativas: ÁREA, VA, D1, D2, D5, D6, D8 e D9. O sinal das variáveis, em sua maioria, está de acordo com o esperado. O valor calculado para o teste da razão de verossimilhança foi altamente significativo, ou seja, existe uma relação significativa entre a variável dependente e as demais variáveis explicativas (Tabela 4).

Tabela 4. Resultados do modelo logit para todos os municípios mineiros, ano 2005

\begin{tabular}{ccccc}
\hline Variável & Coeficiente & Erro padrão & Teste Z & Valor-p \\
\hline CONSTANTE & $-0,1493^{\text {NS }}$ & 0,3345 & $-0,4463$ & 0,6554 \\
AREA & $0,000005^{* *}$ & 0,0000013 & 3,9982 & 0,0001 \\
VA & $0,0000001^{* * *}$ & 0,00000004 & 3,9982 & 0,0001 \\
ANALF & $-0,0081^{\text {NS }}$ & 0,0163 & $-0,4965$ & 0,6195 \\
PO & $-0,000002^{\text {NS }}$ & 0,000004 & $-0,5507$ & 0,5818 \\
D1 & $-0,9077^{* *}$ & 0,4118 & $-2,2044$ & 0,0275 \\
D2 & $-0,9744^{* *}$ & 0,4438 & $-2,1954$ & 0,0281 \\
D3 & $-0,6457^{\text {NS }}$ & 0,4125 & $-1,5656$ & 0,1175 \\
D4 & $0,2652^{\text {NS }}$ & 0,2760 & 0,9608 & 0,3367 \\
D5 & $-1,5626^{* *}$ & 0,6436 & $-2,4279$ & 0,0152 \\
D6 & $-1,0584^{* * *}$ & 0,3805 & $-2,7814$ & 0,0054 \\
D7 & $-0,52^{\text {NS }}$ & 0,3629 & $-1,4328$ & 0,1519 \\
D8 & $-0,5196^{* *}$ & 0,2486 & $-2,0895$ & 0,0367 \\
D9 & $-1,7561^{* * *}$ & 0,3992 & $-4,3995$ & 0,0000 \\
D10 & $-0,92^{\text {NS }}$ & 0,5761 & $-1,5969$ & 0,1103 \\
D11 & $-0,382^{\text {NS }}$ & 0,2906 & $-1,3146$ & 0,1887 \\
\hline Razão de Versossimilhança & 108,398 & & & 0,0000 \\
Obs. com (Y=1)=366 & & & & \\
Obs com (Y=0)=487 & & & & \\
$R^{2}$ Mc Fadden & 0,093 & & & \\
\hline
\end{tabular}

Fonte: Resultados da Pesquisa.

*** significativo a $1 \%$;* significativo a $5 \%$; *significativo a $10 \%$; NS não significativo. 
Sobre as qualidades estatísticas do modelo, observou-se que esse apresentou um poder de previsão de 65,30\% (Tabela 5). Considerando somente as previsões de os municípios receberem o ICMS Ecológico, esse índice é de 37,43\%. Quanto ao não recebimento do ICMS Ecológico, este valor é de $86,24 \%$.

Tabela 5 Previsões certas e erradas do modelo logit estimado

\begin{tabular}{lccc}
\hline & \multicolumn{3}{c}{ Equação estimada } \\
& $\mathrm{Y}=0$ & $\mathrm{Y}=1$ & Total \\
\hline $\mathrm{P}(\mathrm{Y}=1)<=0,50$ & 420 & 229 & 649 \\
$\mathrm{P}(\mathrm{Y}=0)>0,50$ & 67 & 137 & 204 \\
Total & 487 & 366 & 853 \\
Correta & 420 & 137 & 557 \\
\%Correta & 86,24 & 37,43 & 65,30 \\
\%Incorreta & 13,76 & 62,57 & 34,70 \\
\hline
\end{tabular}

Fonte: Resultados da Pesquisa.

A interpretação dos coeficientes é difícil no modelo logit e foi feita através dos efeitos marginais ${ }^{15}$ das variáveis (Tabela 6). Em relação à variável "área", o efeito marginal sobre a probabilidade de o município pertencer à categoria de receptores do ICMS Ecológico foi positivo, conforme esperado. Um aumento em 1.000 hectares na área, mantidas as demais variáveis cons$\operatorname{tantes}^{16}$, faz com que a probabilidade do recebimento do ICMS Ecológico fique aumentada em apenas 0,127 pontos percentuais. Assim, municípios maiores têm maior probabilidade de recebimento, pois eles possuem maior área sujeita a transformação em áreas de proteção.

Com relação à variável "valor adicionado", esperava-se que o seu efeito marginal fosse negativo, pois municípios mais ricos possuem geralmente mais áreas degradadas, dificultando o recebimento do ICMS Ecológico,

15 Conforme discutido na metodologia, o efeito marginal das variáveis explicativas no modelo logit não é constante. Os efeitos marginais foram calculados no ponto médio da amostra. Com relação às variáveis qualitativas, o cálculo foi obtido através da equação (9), enquanto para as variáveis contínuas utilizou-se a equação (8).

16 Para o cálculo do efeito marginal, esta pressuposição é feita para todas as variáveis. 
o que não foi confirmado pelos resultados. Uma explicação possível para este comportamento é que grandes municípios mineiros como Belo Horizonte, Betim, Contagem, Uberlândia, Ipatinga, Uberaba, Juiz de Fora, Itabira, Nova Lima e Ouro Preto, que são municípios que geram um alto valor adicionado, possuem em seu território sistemas de tratamento ou disposição final de lixo urbano e tratamento de esgoto sanitário, recebendo assim um montante de ICMS Ecológico. O sinal positivo encontrado significa que um aumento em $\mathrm{R} \$ 1000,00$ no valor adicionado faz com que a probabilidade do recebimento do ICMS Ecológico fique aumentada em apenas 0,0032 pontos percentuais.

Tabela 6. Efeito marginal para as variáveis selecionadas

\begin{tabular}{ccccc}
\hline Variável & Efeito Marginal & Erro padrão & Teste Z & Valor-p \\
\hline AREA & $0,00000127^{* * *}$ & 0,0000 & 4,00 & 0,000 \\
VA & $0,000000032^{* * *}$ & 0,0000 & 3,54 & 0,000 \\
ANALF & $-0,002^{\text {NS }}$ & 0,00407 & $-0,50$ & 0,619 \\
PO & $-0,00000061^{\text {NS }}$ & 0,0000 & $-0,55$ & 0,582 \\
D1 & $-0,2231^{* *}$ & 0,0975 & $-2,29$ & 0,022 \\
D2 & $-0,2387^{* *}$ & 0,1037 & $-2,30$ & 0,021 \\
D3 & $-0,1596^{\mathrm{NS}}$ & 0,1011 & $-1,58$ & 0,115 \\
D4 & $0,0612^{\text {NS }}$ & 0,0631 & 0,97 & 0,332 \\
D5 & $-0,3624^{* * *}$ & 0,1229 & $-2,95$ & 0,003 \\
D6 & $-0,2580^{* * *}$ & 0,0878 & $-2,94$ & 0,003 \\
D7 & $-0,1283^{\mathrm{NS}}$ & 0,0897 & $-1,43$ & 0,152 \\
D8 & $-0,1282^{* *}$ & 0,0608 & $-2,11$ & 0,035 \\
D9 & $-0,3963^{* * *}$ & 0,075 & $-5,28$ & 0,000 \\
D10 & $-0,2260^{*}$ & 0,1361 & $-1,66$ & 0,097 \\
D11 & $-0,0938^{\mathrm{NS}}$ & 0,0713 & -132 & 0,188 \\
\hline
\end{tabular}

Fonte: Resultados da Pesquisa.

*** significativo a $1 \% ; * *$ significativo a $5 \%$; *significativo a $10 \%$; NS não significativo. 
As seguintes variáveis dummies: D1, D2, D5, D6, D8 e D9 apresentaram efeitos marginais significativos e negativos, o que significa que se o município pertencer a uma destas mesorregiões (Campos das Vertentes, Central Mineira, Noroeste de Minas, Norte de Minas, Sul e Sudoeste e Triângulo Mineiro e Alto do Paranaíba, respectivamente), a probabilidade de ele pertencer à categoria de receptores do ICMS Ecológico em relação aos municípios da Zona da Mata (mesorregião padrão) diminui, respectivamente, em 22,$31 ; 23,87 ; 36,24 ; 25,80 ; 12,82$ e 39,63 pontos percentuais. Este resultado era esperado, pois, em 2005, 50,35\% dos municípios da Zona da Mata receberam o ICMS Ecológico, enquanto que nas mesorregiões: Campos das Vertentes, Central Mineira, Noroeste de Minas, Norte de minas, Sul e Sudoeste e Triângulo Mineiro e Alto do Paranaíba, este valor foi, respectivamente, de: $30,56 \%, 33,33 \%, 47,37 \%, 29,21 \%, 41,78 \%$ e 36,92\%. Assim, a maior parte dos municípios da Zona da Mata recebe o ICMS Ecológico e os municípios com as mesmas características localizados em outras mesorregiões têm probabilidade menor de receber esse recurso.

As variáveis dummies D3, D4 D7, D10 e D11 foram não significativas (a 5\%), indicando que se o município pertencer, respectivamente, a uma destas mesorregiões (Jequitinhonha, Metropolitana de Belo Horizonte, Oeste de Minas, Vale do Mucuri e Vale do Rio Doce) não há influência na probabilidade de pertencer à categoria de receptores do ICMS Ecológico, comparado com a mesorregião da Zona da Mata.

Em relação à variável que mede o índice de analfabetismo do município, ela se mostrou não significativa. Isto significa que municípios com alta e baixa taxa de analfabetismo têm a mesma probabilidade de receber o ICMS Ecológico. A variável "tamanho da população" também foi não significativa, indicando que ela não é importante como determinante do recebimento do ICMS Ecológico, pois tanto municípios pequenos quanto grandes podem possuir em seu território Unidades de Conservação ou trabalharem com a questão do saneamento ambiental para recebimento dos recursos do ICMS Ecológico.

Assim, as variáveis quantitativas AREA e VA e as variáveis qualitativas D1, D2, D5, D6, D8 e D9 (relacionadas ao município pertencer, respectivamente, às mesorregiões: Campos das Vertentes, Central Mineira, Noroeste de Minas, Norte de Minas, Sul e Sudoeste de Minas e Triângulo Mineiro e Alto do Paranaíba, sendo a Zona da Mata a variável de controle) foram as principais determinantes da probabilidade de o município receber o ICMS Ecológico. 


\title{
4. CONCLUSÕES
}

Com relação aos efeitos redistributivos do ICMS Ecológico, pode-se concluir que ele beneficia mais intensamente os municípios menores, mas seu impacto é pequeno pelo reduzido valor de seu percentual (1\%). Além disso, o impacto do ICMS Ecológico para esses municípios vem perdendo força nos últimos anos com o aumento do numero de municípios recebedores, o que é preocupante em termos do incentivo a novos municípios que queiram se adequar às normas de recebimento deste recurso.

Já a probabilidade de recebimento do ICMS Ecológico varia positivamente com a área dos municípios e o ICMS recebido através do critério valor adicionado. A mesorregião a qual o município pertence também é importante na probabilidade de recebimento deste recurso.

Recomenda-se que haja uma reformulação nos critérios da Lei Robin Hood para que se aumente o percentual de ICMS Ecológico destinado aos municípios mineiros, de forma que não seja comprometida a eficácia de seu estímulo para os municípios se engajarem em ações de preservação.

\section{REDISTRIBUTIVE EFFECTS AND DETERMINANT OF THE ECOLOGICAL VAT RECEIVINGS BY THE CITIES OF MINAS GERAIS - BRAZIL}

\begin{abstract}
The objective of this study was to evaluate the change in the VAT distribution in Minas Gerais caused by the introduction of the ecological criterion. Another goal was to evaluate the determinants of counties on receiving the Ecological VAT. Results showed that distributional effects were small mainly due to the small percentage $(1 \%)$ allocated to counties, benefiting mainly small counties. Besides, Ecological VAT impact is becoming weaker with the increasing number of receiving counties, which raises concerns on lack of incentives to new counties to adapt to receiving regulations.
\end{abstract}

Keywords: Ecological VAT, Minas Gerais, Logit Model. 


\section{REFERÊNCIAS BIBLIOGRÁFICAS}

FJP - FUNDAÇÃO JOÃO PINHEIRO. Disponível em: http://www.fjp. gov.br. Acesso em 11/08/2007.

GRIEG-GRAN, M. Fiscal incentives for biodiversity conservation: The ICMS Ecológico in Brazil. Discussion Paper 00-01 International Institute for Environment and Development, London, UK. 2000.

GUJARATI, D. N. Econometria básica. 4.ed. São Paulo: Campus, 2006. $812 \mathrm{p}$.

IBGE - INSTITUTO BRASILEIRO DE GEOGRAFIA E ESTATÍSTICA. Disponível em: http://www.ibge.gov.br. Acesso em set. 2007.

INEP - Instituto Nacional de Estudos e Pesquisas Educacionais Anísio Teixeira. Disponível em: http://ideb.inep.gov.br/Site/. Acesso em $14 / 01 / 2008$.

LOUREIRO, W. Contribuição do ICMS Ecológico à conservação da biodiversidade no Estado do Paraná. Curitiba, PR, UFPR. 2002. 189f.; Dissertação (Doutorado em Ciências Florestais).

MINAS GERAIS. Lei n ${ }^{\circ} 12.040$, de 28 de dezembro de 1995. Dispõe sobre a distribuição da parcela de receita do produto da arrecadação do ICMS pertencente aos municípios, de que trata o inciso II do parágrafo único do artigo 158 da Constituição Federal, e dá outras providências. Diário Oficial do Estado de Minas Gerais, Belo Horizonte, 1995.

NUNES, L.H.C. ICMS Ecológico: revisão crítica dos critérios de distribuição do produto da arrecadação dos impostos pertencentes aos municípios. Belo Horizonte, MG Fundação João Pinheiro. 2003. 143f.; Dissertação (Mestrado em Administração Pública). 


\section{ANEXO A}

Sistemática do cálculo do índice de participação dos municípios mineiros no ICMS Ecológico

De acordo com a Lei 13.803/00 (Lei Robin Hood), o ICMS Ecológico é decomposto em duas partes. Uma diz respeito ao saneamento ambiental $\left(\right.$ ICMS $\left.^{\mathrm{A}}\right)$ e outra a Unidade de Conservação $\left(\mathrm{ICMS}^{\mathrm{C}}\right)$. A soma das duas partes,

$$
\mathrm{ICMS}^{\mathrm{A}}+\mathrm{ICMS}^{\mathrm{C}}=\mathrm{x} * \mathrm{ICMS}_{\mathrm{T}}
$$

em que $x$ é a parcela alocada para fins de preservação do meio ambiente (a partir de 1998, x=0,0025) e $\mathrm{ICMS}_{\mathrm{T}}$ é o total arrecadado do ICMS.

Em seguida, define-se I, índice de participação do município j no total do ICMS Ecológico, como sendo:

$$
\mathrm{I}_{\mathrm{j}}=\mathrm{ISA}_{\mathrm{j}}+\mathrm{IC}_{\mathrm{j}}
$$

em que ISA $\mathrm{j}_{\mathrm{j}}$ o índice de saneamento ambiental do município j e IC é o índice de conservação do município j.

$\mathrm{O}$ valor obtido pelo município do ICMS é dado por $\mathrm{I}_{\mathrm{j}} * \mathrm{x} * \mathrm{ICMS}_{\mathrm{T}}$.

$\mathrm{O}$ Índice de Saneamento Ambiental pode ser obtido pela seguinte expressão:

$\mathrm{ISA}_{\mathrm{j}}=(1 / \mathrm{N}) * \mathrm{ICMS}^{\mathrm{A}}$, respeitando a condição $\operatorname{ICMS}^{\mathrm{A}} \leq(0,5 * \mathrm{x} *$ $\left.\mathrm{ICMS}_{\mathrm{T}}\right)$ e $\left(\mathrm{ISA}_{\mathrm{j}} * \mathrm{x} * \mathrm{ICMS}_{\mathrm{T}}\right) \leq \mathrm{CP}_{\mathrm{j}}$

em que $\mathrm{N}$ é o número de municípios que possuem unidades autorizadas pelo COPAM para tratamento de esgoto ou para tratamento de lixo e $\mathrm{CP}_{\mathrm{j}}$ é o custo de implantação da unidade de tratamento de esgoto e/ou lixo do município j.

O Índice de Conservação para o município j é:

$\mathrm{IC}_{\mathrm{j}}=\mathrm{FCM}_{\mathrm{j}} / \sum_{\mathrm{j}} \mathrm{FCM}_{\mathrm{j}}$

em que FCM é o fator de conservação do município j.

A expressão do fator de conservação, por sua vez, pode ser obtida: 


$$
\mathrm{FCM}_{\mathrm{j}}=\left(\mathrm{AEuc}_{\mathrm{j}} / \mathrm{Am}_{\mathrm{j}}\right)^{*} \mathrm{~F}_{\mathrm{q}}
$$

em que $\mathrm{AEuc}_{\mathrm{i}}$ é a área equivalente ocupada pelas Unidades de Conservação no município j; $A_{j}$ é a área total do município j e $F_{q}$ é um fator de qualidade que assume valores de $0,1 \mathrm{a} 1$. Esse fator é relativo à qualidade física da área, plano de manejo, infra-estrutura, entorno protetor, estrutura de proteção e fiscalização etc. Em Minas Gerais, este fator de qualidade assume valor igual a um.

É importante observar que a área equivalente ocupada pelas Unidades de Conservação no município j pode ser estruturada da seguinte maneira:

$$
\begin{aligned}
\text { AEuc }_{j} & =\mathrm{EE}_{\mathrm{j}}+\mathrm{RB}_{\mathrm{j}}+\mathrm{APAI}_{\mathrm{j}}+0,9 \mathrm{PAQ}_{\mathrm{j}}+0,9 \mathrm{RPPN}_{\mathrm{j}}+0,7 \mathrm{FLO}_{\mathrm{j}}+0,5 \mathrm{AI}_{\mathrm{j}} \\
+ & 0,1 \mathrm{APE}_{\mathrm{j}}+0,1 \mathrm{ZVS}_{\mathrm{j}}+0,025 \mathrm{APAII}_{\mathrm{j}}
\end{aligned}
$$

em que $E_{\mathrm{j}}$ é a área da estação ecológica no município $\mathrm{j} ; \mathrm{RB}_{\mathrm{j}}$ é a área da reserva biológica no município $\mathrm{j} ; \mathrm{ZVS}_{\mathrm{j}}$ é a zona de vida silvestre no município j; $P A Q_{j}$ é a área ocupada com parques no município j; RPPN $\mathrm{e}_{\mathrm{j}}$ a área ocupada por reserva particular do patrimônio natural no município j; $\mathrm{FLO}_{\mathrm{j}}$ é a área ocupada com floresta nacional, estadual ou municipal no município $\mathrm{j} ; \mathrm{AI}_{\mathrm{j}}$ é a área ocupada com reserva indígena no município $\mathrm{j}$; $\mathrm{APE}$ é a área de proteção especial no município j; APAI é a área de proteção ambiental que dispõe de zoneamento ecológico-econômico no município j; e, APAII $\mathrm{j}_{\mathrm{j}}$ a a área de proteção ambiental que não dispõe de zoneamento ecológico-econômico no município j. 


\begin{tabular}{|c|c|c|c|c|c|c|c|c|c|c|c|c|c|c|c|c|}
\hline \multirow{10}{*}{ 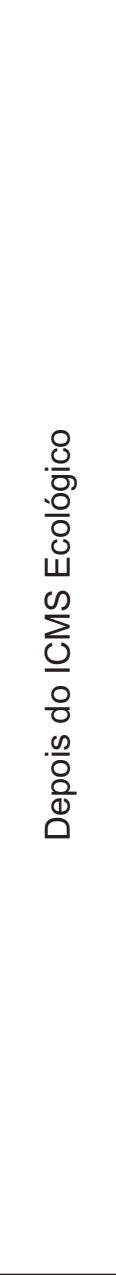 } & 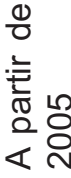 & \begin{tabular}{|l}
$\infty$ \\
0 \\
0 \\
1
\end{tabular} & $\frac{0}{\frac{0}{\sigma}}$ & $\begin{array}{l}10 \\
10\end{array}$ & 0 & 0 & O & $\begin{array}{l}\bar{N} \\
\sim\end{array}$ & $\stackrel{\circ}{\sim}$ & $\begin{array}{l}0 \\
\sim \\
\sim\end{array} \mid$ & $\stackrel{\circ}{\sim}$ & $\begin{array}{l}0 \\
\sigma\end{array}$ & $\begin{array}{l}0 \\
-\end{array}$ & $\begin{array}{l}0 \\
\mathfrak{N}\end{array}$ & $\stackrel{\circ}{N}$ & 응 \\
\hline & ষ্ণ & $\begin{array}{l}\infty \\
0 \\
0 \\
0 \\
0 \\
0\end{array}$ & $\frac{0}{\frac{0}{\sigma}}$ & $\begin{array}{l}10 \\
10\end{array}$ & $\begin{array}{l}\infty \\
0 \\
0 \\
0 \\
0\end{array}$ & $\mid \begin{array}{l}\text { ঠ } \\
\text { ○ } \\
0\end{array}$ & 어 & $\begin{array}{c}\bar{N} \\
\sim\end{array}$ & $\stackrel{\circ}{\sim}$ & $\begin{array}{l}0 \\
\text { N }\end{array}$ & $\stackrel{\circ}{\circ}$ & $\begin{array}{l}0 \\
5\end{array}$ & $\begin{array}{l}0 \\
-\end{array}$ & $\begin{array}{l}0 \\
N\end{array}$ & $\stackrel{0}{\sim}$ & 응 \\
\hline & $\begin{array}{l}\text { @ } \\
\text { ㅇ }\end{array}$ & $\begin{array}{l}6 \\
0 \\
0 \\
0 \\
\Omega \\
N\end{array}$ & $\frac{0}{\frac{0}{6}}$ & $\begin{array}{l}10 \\
10\end{array}$ & $\begin{array}{l}0 \\
0 \\
0 \\
0\end{array}$ & $\mid \begin{array}{l}\infty \\
0 \\
0 \\
0 \\
0\end{array}$ & 엉 & $\begin{array}{l}\bar{N} \\
\sim\end{array}$ & $\stackrel{0}{N}$ & $\begin{array}{c}0 \\
\sim \\
\sim\end{array} \mid$ & $\stackrel{\circ}{\circ}$ & $\begin{array}{l}0 \\
-\end{array}$ & $\begin{array}{l}0 \\
-\end{array}$ & $\begin{array}{l}0 \\
N\end{array}$ & $\stackrel{\circ}{N}$ & 음 \\
\hline & $\begin{array}{l}\text { N } \\
\stackrel{\mathrm{O}}{ }\end{array}$ & 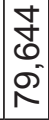 & $\frac{0}{\frac{0}{\sigma}}$ & $\begin{array}{l}10 \\
10\end{array}$ & \begin{tabular}{l}
\multirow{J}{*}{} \\
0 \\
0 \\
0
\end{tabular} & $\begin{array}{l}N \\
\vdots \\
0 \\
0\end{array}$ & 0 & $\begin{array}{c}\bar{\tau} \\
\tilde{N}\end{array}$ & $\stackrel{\circ}{N}$ & $\begin{array}{c}0 \\
\text { Ni }\end{array} \mid$ & $\stackrel{-}{\circ}$ & $a_{-}^{0}$ & $\stackrel{0}{-}$ & $\begin{array}{l}0 \\
\cdots\end{array}$ & $\stackrel{\circ}{\sim}$ & 음 \\
\hline & ঠ্ণ & $\begin{array}{l}\tilde{N} \\
0 \\
0 \\
N\end{array}$ & $\frac{0}{\frac{0}{\sigma}}$ & $\begin{array}{l}10 \\
10\end{array}$ & $\begin{array}{l}N \\
\tilde{n} \\
0 \\
0\end{array}$ & $\mid \begin{array}{l}0 \\
0 \\
0 \\
0 \\
0\end{array}$ & 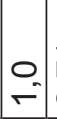 & $\begin{array}{l}\bar{N} \\
\tilde{N}\end{array}$ & $\stackrel{0}{i}$ & $\begin{array}{l}0 \\
\text { Ni }\end{array} \mid$ & $\stackrel{0}{\circ}$ & $\begin{array}{l}0 \\
-\end{array}$ & 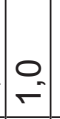 & $\begin{array}{l}0 \\
\text { N }\end{array}$ & $\stackrel{\circ}{\sim}$ & 은 \\
\hline & $\begin{array}{l}\text { ᄋ } \\
\text { ㅇ }\end{array}$ & $\begin{array}{l}\widetilde{N} \\
0 \\
\Omega \\
\end{array}$ & $\frac{5}{\sigma}$ & $\begin{array}{l}10 \\
10 \\
\end{array}$ & $\begin{array}{l}10 \\
0 \\
0 \\
0\end{array}$ & $\begin{array}{l}\mathcal{N} \\
0 \\
0\end{array}$ & $\begin{array}{c}0 \\
-\end{array}$ & $\begin{array}{l}\bar{N} \\
\sim \\
\sim\end{array}$ & $\stackrel{0}{i}$ & $\begin{array}{l}0 \\
\text { N }\end{array} \mid$ & $\stackrel{0}{-}$ & $0_{-}^{\circ}$ & $a_{-}^{\circ}$ & ì & $\stackrel{\circ}{\sim}$ & 음 \\
\hline & ஓ) & 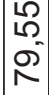 & $\underset{\sigma}{\sigma}$ & $\begin{array}{l}10 \\
10\end{array}$ & $\begin{array}{l}9 \\
0 \\
0\end{array}$ & \begin{tabular}{l}
\multirow{0}{0}{} \\
0
\end{tabular} & $\begin{array}{l}0 \\
-1\end{array}$ & $\bar{\sim}$ & $\stackrel{\circ}{\sim}$ & $\begin{array}{l}0 \\
\sim \\
\sim\end{array} \mid$ & $\stackrel{0}{r}$ & $\begin{array}{l}0 \\
\sigma\end{array}$ & $\begin{array}{l}0 \\
-\end{array}$ & $\begin{array}{l}0 \\
N \\
N\end{array}$ & $\stackrel{0}{N}$ & 음 \\
\hline & $\begin{array}{l}\text { ᄋ̊ } \\
\text { ᄋ }\end{array}$ & $\mid \begin{array}{l}\sigma \\
+ \\
\sigma^{\circ}\end{array}$ & $\frac{F}{0}$ & $\begin{array}{l}10 \\
10\end{array}$ & $\frac{5}{0}$ & $\mid \begin{array}{l}0 \\
0 \\
0 \\
0\end{array}$ & $\begin{array}{c}0 \\
-\end{array}$ & $\begin{array}{l}\bar{\tau} \\
\tilde{N}\end{array}$ & $\stackrel{\circ}{\sim}$ & $\begin{array}{l}0 \\
\text { Ni }\end{array} \mid$ & $\stackrel{0}{\circ}$ & $0_{-}^{\circ}$ & $\stackrel{0}{\circ}$ & $\begin{array}{c}0 \\
\text { N }\end{array}$ & $\stackrel{\circ}{\sim}$ & 음 \\
\hline & 命 & 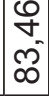 & 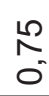 & $\begin{array}{l}10 \\
10\end{array}$ & $\frac{\infty}{\sigma_{0}^{\prime}}$ & $\begin{array}{l}\infty \\
0 \\
0 \\
0\end{array}$ & $\left|\begin{array}{c}\hat{0} \\
0\end{array}\right|$ & 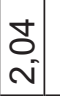 & $\stackrel{m}{m}$ & 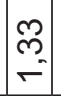 & $\begin{array}{l}0 \\
0 \\
0\end{array}$ & $\begin{array}{l}1 \\
0 \\
0\end{array}$ & $\begin{array}{l}\hat{0} \\
0\end{array}$ & $\stackrel{m}{m}$ & $\stackrel{m}{m}$ & 응 \\
\hline & ஜ & $\begin{array}{l}0 \\
0 \\
\infty \\
\infty \\
\infty\end{array}$ & $\stackrel{0}{\sigma}$ & $\begin{array}{l}10 \\
10\end{array}$ & $\begin{array}{l}\stackrel{\Upsilon}{N} \\
0\end{array}$ & व & $\left|\begin{array}{c}m \\
m \\
0\end{array}\right|$ & $\begin{array}{l}1 \\
0 \\
0\end{array}$ & $\begin{array}{l}\hat{0} \\
0\end{array}$ & $\left|\begin{array}{l}0 \\
0 \\
0\end{array}\right|$ & $\begin{array}{c}m \\
m \\
0 \\
0\end{array}$ & $\begin{array}{c}m \\
m \\
0 \\
0\end{array}$ & $\begin{array}{l}m \\
m \\
0\end{array}$ & $\begin{array}{l}1 \\
0 \\
0\end{array}$ & $\begin{array}{l}\hat{0} \\
0\end{array}$ & 음 \\
\hline 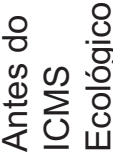 & Љூ & \begin{tabular}{|l|} 
\\
$\sigma$ \\
ల్ \\
$\sigma$
\end{tabular} & $\frac{F}{\sigma}$ & $\begin{array}{l}10 \\
10\end{array}$ & $\begin{array}{l}0 \\
\text { m. } \\
0\end{array}$ & $\mid \begin{array}{l}\mathcal{N} \\
\tilde{0}\end{array}$ & & & & & & & & & & 응 \\
\hline
\end{tabular}

